\title{
CÁLCIO EXÓGENO COMO BIOMARCADOR DO USO DE VIA METABÓLICA ALTERNATIVA POR BIOMPHALARIA SPP (Mollusca, Planorbidae)
}

\section{Luciana Damacena Silva}

Universidade Estadual de Goiás, Campus de Ciências Exatas e Tecnológicas - Henrique Santillo, Curso de Ciências Biológicas. Anápolis, Goiás, Brasil, luciana.silva@ueg.br

\section{Sueli Martins De freitas Alves}

Universidade Estadual de Goiás, Campus de Ciências Exatas e Tecnológicas - Henrique Santillo, Curso de Engenharia Agrícola, Anápolis, Goiás, Brasil, suelifreitas@ueg.br

\section{Kamila Souto Leichtweis}

Universidade Estadual de Goiás, Campus de Ciências Exatas e Tecnológicas - Henrique Santillo, Anápolis, Goiás, Brasil, kamilaleichtweis@hotmail.com

\section{Daniela Braz Dos Santos}

Universidade Federal de Goiás, Instituto de Patologia Tropical e Saúde Pública, Goiânia, Goiás, Brasil, danielabrazdossantos@gmail.com

\section{Ana Maria de Castro}

Universidade Federal de Goiás, Instituto de Patologia Tropical e Saúde Pública, Goiânia, Goiás, Brasil, amaria.ana@gmail.com

\section{Clélia Christina Mello-Silva}

FIOCRUZ, Instituto Oswaldo Cruz /IOC /FIOCRUZ, Rio de Janeiro-RJ, Brasil, cleliachristinamellosilva@gmail.com

\section{Karine Borges Machado}

Universidade Federal de Goiás, Instituto de Ciências Biológicas, Goiânia, Goiás, Brasil, machadokb@gmail.com

\section{Hânstter Hallison Alves Rezende}

Universidade Federal de Goiás, Unidade Acadêmica de Ciências da Saúde - CISAU, Jataí, Goiás, Brasil, hanstter@gmail.com

\section{José Clecildo Barreto Bezerra}

Universidade Federal de Goiás, Instituto de Patologia Tropical e Saúde Pública, Goiânia, Goiás, Brasil, clecildobarreto@gmail.com

Resumo: Caramujos do gênero Biomphalaria são hospedeiros do parasito Schsitosoma mansoni, bem como, bioindicadores de qualidade ambiental. Esses caramujos usam carboidratos como fonte primária de energia e aminoácidos na síntese de "novo" de glicose, pela gliconeogênese. A concentração do $\mathrm{Ca}^{2+}$ exógeno afeta o metabolismo energético dos caramujos. Este estudo avaliou o 
uso de uma via metabólica alternativa por Biomphalaria tenagophila e B. glabrata expostas a 20, 40, 60,80 e $100 \mathrm{mg} / \mathrm{L}$ de $\mathrm{CaCO}_{3}$, por 30 dias, a partir das concentrações de proteína totais, ureia, ácido úrico e creatinina, obtidas utilizando espectrofotômetro. Para cada grupo, foram utilizados quinze caramujos, dos quais foram obtidos amostras compostas de hemolinfa. De maneira geral, houve aumento de proteínas totais e redução de ureia, em ambas as espécies, expostas às maiores concentrações de $\mathrm{CaCO}_{3}$, comparado ao controle. O ácido úrico também aumentou para as duas espécies nas maiores concentrações de $\mathrm{CaCO}_{3}$. Maiores valores de creatinina foram observados em $B$. tenagophila e $B$. glabrata expostas, respectivamente, a 20 e $40 \mathrm{mg} \mathrm{CaCO}$. O uso de proteínas como substrato energético foi observado principalmente na exposição a $20 \mathrm{mg}$ de $\mathrm{CaCO}_{3}$, além de particularidades na regulação de mecanismos fisiológicos pelas espécies como hospedeiras de $S$. mansoni.

Palavras-chave: Carbonato de cálcio, moluscos hospedeiros da esquistossomose, produtos nitrogenados, vias metabólicas alternativas.

\section{THE EXOGENOUS CALCIUM AS A BIOMARKER OF THE USE OF ALTERNATIVE METABOLIC VIA BY Biomphalaria spp (Mollusca, Planorbidae)}

Abstract: Snails of the genus Biomphalaria are hosts of the parasite Schistosoma mansoni, as well as bioindicators of environmental quality. These snails use carbohydrates as the primary source of energy and amino acids in the "new" synthesis of glucose by gluconeogenesis. The concentration of exogenous $\mathrm{Ca}^{2+}$ affects the energetic metabolism of these snails. This study evaluated the use of an alternative metabolic pathway by Biomphalaria tenagophila and B. glabrata exposed to 20, 40,60, 80 and $100 \mathrm{mg} / \mathrm{L} \mathrm{CaCO}_{3}$ for 30 days. From each group, samples of hemolymph of fifteen snails from each group were used assess the level of total protein, urea, uric acid and creatinine by spectrophotometry. In general, there was an increase of total proteins and reduction of urea, in both species, exposed to the higher concentrations of $\mathrm{CaCO}_{3}$, when compared to the control. Uric acid also increased for both species in the highest concentrations of $\mathrm{CaCO}_{3}$. Higher creatinine values were observed in B. tenagophila and B. glabrata exposed, respectively, to 20 and $40 \mathrm{mg} \mathrm{CaCO}$. The use of proteins as energetic substrate was observed mainly in the exposure to $20 \mathrm{mg}$ of $\mathrm{CaCO}_{3}$, besides particularities in the regulation of physiological mechanisms were observed in these hosts of $S$. mansoni.

Keywords: Calcium carbonate, host snails for schistosomiasis, nitrogenated products, alternative metabolic pathways.

\section{INTRODUÇÃO}

Os caramujos do gênero Biomphalaria são importantes hospedeiros intermediários no ciclo do parasito trematódeo Schistosoma mansoni (Sambon, 1907), agente etiológico da esquistossomose mansônica (Lardans \& Dissous, 1998). No Brasil, a manutenção do ciclo deste parasito está condicionada à presença de caramujos das espécies Biomphalaria glabrata (Say, 1818), Biomphalaria tenagophila (Orbigny, 1835) e B. straminea (Dunker, 1848) (Paraense, 2001), em ambientes dulcícolas.

A esquistossomose é uma doença infecciosa negligenciada, que ocorre em regiões tropicais e subtropicais (Elbaz \&
Esmat, 2013). Esta doença tem prevalência elevada, sendo altamente debilitante, com ocorrência na África, Ásia e América do Sul (Gray et al., 2010; World Health Organization, 2012). Nessas regiões, a infecção e re-infecção se mantém, em pessoas que vivem em condições precárias de higiene, saneamento insatisfatórias ou ausentes, e onde a exposição a fontes de água contaminadas é inevitável (Elbaz \& Esmat, 2013), bem como, a ocorrência da esquistossomose estando relacionada com a distribuição dos caramujos hospedeiros intermediários (Scholte et al., 2012).

Para o controle dos caramujos Biomphalaria a Organização Mundial da Saúde (OMS) recomenda a niclosamida, comercialmente denominada Bayluscide ${ }^{\circledR}$. Este agente moluscicida é eficaz em todas as fases de desenvolvimento do caramujo, ou seja, embriões, recém-eclodidos e caramujos 
adultos. Entretanto, apresenta toxicidade a outros animais aquáticos e plantas, baixa solubilidade, tanto em solventes orgânicos quanto em água e, ainda, possui alto custo (King \& Bertsch, 2015).

Nesse sentido, as buscas para o controle da esquistossomose continuam, seja, através de estudos envolvendo o caramujo hospedeiro (Pinheiro et al., 2009; Tunholi et al., 2010) ou da interação parasito-hospedeiro (Becker, 1980). O parasitismo intramolusco provoca desequilíbrio metabólico, especialmente em suas reservas glicídicas. Os gastrópodes pulmonados têm seu metabolismo energético baseado em carboidratos, os quais são armazenados sob a forma de polissacarídeos (Joosse, 1988). Uma vez exauridos os depósitos de carboidratos, que resulta a um estado de inanição, seu equilíbrio metabólico tenta ser restabelecido por meio da metabolização de proteínas e aminoácidos (Becker, 1980; Pinheiro et al., 2009). Nessas condições metabólicas, os aminoácidos podem ser utilizados para a síntese de "novo" de glicose, pela gliconeogênese (Nelson \& Cox, 2011).

A utilização de proteínas como substrato, na gliconeogênese, eleva as concentrações hemolinfáticas de ureia, ácido úrico e amônia, que são seus produtos nitrogenados de excreção (Pinheiro et al., 2009). Em invertebrados, a creatinina surge como produto intermediário do ciclo da ureia ou do catabolismo do fosfato de creatina (Allen et al., 2011; Hiong et al., 2005).

Os substratos para produção de energia, utilizando via aeróbia ou anaeróbia por caramujos gastrópodes, são dependentes das condições do habitat (Livingstone \& de Zwaan, 1983), tendo influência a hidrografia, temperatura, $\mathrm{pH}$, concentrações de compostos orgânicos e inorgânicos (Barbosa \& Barbosa, 1994). O íon cálcio é um composto inorgânico descrito como fator limitante na distribuição e adaptação desses animais no ambiente, além de apresentar importância em vários aspectos fisiológicos e metabólicos (Becker, 1980). Em processos metabólicos atua como solução tampão na hemolinfa. Por exemplo, ocorre mobilização de carbonato de cálcio, excretados ou metabolizados na concha para hemolinfa em resposta à liberação de ácidos orgânicos que podem ser tóxicos para o caramujo (Becker, 1980; Davies \& Erasmus, 1984; Liebsch \& Becker, 1990).

No epitélio intestinal e cutâneo dos moluscos o cálcio é absorvido passivamente, quando concentrações no ambiente está em nível de $80 \mathrm{mg} / \mathrm{L}$ (Greenaway, 1971). Em locais onde o cálcio é limitado a menos de $50 \mathrm{mg} / \mathrm{L}$, o transporte epitelial desse íon ocorre com gasto de energia, e os moluscos tem respiração cutânea aumentada e diminuição na motilidade (Dalesman et al., 2011).

O conhecimento da influência do cálcio no metabolismo energético dos caramujos do gênero Biomphalaria poderá auxiliar no desenvolvimento de novos compostos com propriedades moluscicidas. Nessa perspectiva, o objetivo deste estudo foi avaliar a influência de diferentes concentrações de $\mathrm{CaCO}_{3}$ nas concentrações de proteína totais, ureia, ácido úrico e creatinina, na hemolinfa de $B$. tenagophila e $B$. glabrata.

\section{Material e métodos}

Foram utilizados caramujos das espécies B. glabrata, cepa pigmentada de Belo Horizonte/MG e B. tenagophila, cepa de Joinville/SC, procedentes do Laboratório de Malacologia do Instituto de Patologia Tropical e Saúde Pública. Os animais foram mantidos em aquários de polietileno, contendo $8 \mathrm{~L}$ de água filtrada e desclorada, $\mathrm{pH}$ neutro a alcalino e temperatura $25^{\circ} \mathrm{C}\left( \pm 1^{\circ} \mathrm{C}\right)$. Os aquários foram monitorados diariamente e higienizados uma vez por semana. Os caramujos foram alimentados ad libitum, com folhas frescas de alface (Lactuca sativa L.), previamente higienizada em água corrente. Este trabalho foi submetido e aprovado pela Comissão de Ética no Uso de Animais CEUA-PRPPG-UFG, com protocolo $n$. 083/12.

\section{COMPOSIÇÃo dOS GRUPOS DE ESTUdO}

Para a realização deste estudo, foram utilizados 120 exemplares para cada espécie. Os caramujos foram expostos as diferentes concentrações de $\mathrm{CaCO}_{3}(20,40,60,80$ e 100 $\mathrm{mg} / \mathrm{L})$, por 30 dias.

Os caramujos foram retirados dos aquários de manutenção aleatoriamente, alternando entre indivíduos presentes na superfície e fundo do aquário. Na sequência foram acondicionadas em caixas plásticas contendo soluções (água filtrada e desclorada $+\mathrm{CaCO}_{3}$ ) nas concentrações 20, 40,60, 80 e $100 \mathrm{mg} / \mathrm{L}$ de $\mathrm{CaCO}_{3}$. Em cada caixa devidamente identificada com o respectivo tratamento, o volume final da solução foi de $4 \mathrm{~L}$. Semanalmente, as caixas eram lavadas e as concentrações de $\mathrm{CaCO}_{3}$ renovadas. O grupo controle contou com caramujos alocados apenas em água filtrada e desclorada (água fornecida pela Companhia Saneamento de Goiás S. A. - SANEAGO, armazenada em caixa d'água com capacidade para 1000 L no ambiente interno do laboratório de malacologia, para promover a decantação de cloro), no mesmo volume das soluções de $\mathrm{CaCO}_{3}$.

\section{Extração da Hemolinfa}

As conchas dos caramujos foram higienizadas e secas com papel absorvente para a extração da hemolinfa. Em seguida uma 
agulha $0,7 \times 25$ (tipo 22G 1) acoplada a uma seringa de $1 \mathrm{~mL}$, foi introduzida na cavidade pericárdica de cada indivíduo para a retirada da hemolinfa. Esta etapa foi realizada sob um microscópio estereoscópico Zeiss STEMI DV4. A hemolinfa coletada foi acondicionada em microtubos de $1 \mathrm{~mL}$ e mantidas em gelo. Para cada grupo, foram sacrificados 15 caramujos com 30 dias de exposição ao $\mathrm{CaCO}_{3}$. Foram obtidas amostras compostas de hemolinfa, devido à pequena quantidade de amostra obtida por caramujo. Cada amostra continha hemolinfa de 5 indivíduos do mesmo grupo experimental. Os moluscos colocados a mais nas caixas experimentais foram mantidos por trinta dias em uma caixa contendo água filtrada e desclorada, posteriormente devolvidos aos aquários de manutenção.

\section{Dosagem da CONCENTRAÇÃo DE PROTEÍNAs TOTAIS, UREIA, ÁCIDO ÚRICO E CREATININA}

As concentrações de proteínas totais, ureia e ácido úrico foram obtidas utilizando kits específicos da marca LABTEST ${ }^{\circledR}$. Para a dosagem da creatinina utilizou-se o kit da marca DOLES ${ }^{\circledR}$, método Jaffé modificado (Henry, 1996). Todos os materiais analisados foram lidos em espectrofotômetro (BIO 2000 $\left.{ }^{\odot}\right)$, da marca BIOPLUS ${ }^{\circledR}$, em $545 \mathrm{~nm}$.

\section{AnÁlise de Dados}

Para avaliar se existem diferenças nas concentrações dos produtos do metabolismo de acordo com o tipo de tratamento, foi utilizada uma análise de variância fatorial (Anova). Cada réplica foi composta pela quantidade de proteínas totais, ureia, ácido úrico e creatinina extraídos para cinco indivíduos submetidos à mesma dosagem de $\mathrm{CaCO}_{3}$. Essa estratégia foi adotada devido à baixa quantidade de hemolinfa extraída dos caramujos, que impossibilitou o uso de cada indivíduo como uma réplica. Porém a seleção dos indivíduos foi realizada de forma aleatória dentro de cada tratamento. Nessa análise, a concentração de proteínas, ureia, ácido úrico e creatinina foram consideradas como as variáveis respostas quantitativas enquanto o tipo de tratamento como o preditor categórico. A Anova foi realizada separadamente de acordo com o tipo de produto do metabolismo e espécie avaliada. Dessa forma, o valor de significância foi estabelecido de acordo com critério de Bonferroni para múltiplos testes (a / número de testes, ver Legendre \& Legendre, 1998), sendo considerados significativos valores de $P<0,01$. Nas situações em que os resultados da Anova foram significativos, foi realizado o teste de Tukey para verificar entre quais tratamentos essas diferenças ocorrem. Todos os testes foram realizados no programa $\mathrm{R}$ (Core Team $R$, 2016) através das funções "aov" e "TukeyHSD" do pacote Vegan (Oksanen et al., 2017).

\section{Resultados e Discussão}

As concentrações de proteínas totais, ureia, ácido úrico e creatinina diferem entre os tratamentos para as duas espécies avaliadas (Tab. 1). Para B. tenagophila, em média, a concentração de proteínas totais aumentou nos caramujos expostos a 40 e $80 \mathrm{mg}$ de $\mathrm{CaCO}_{3}$ (Fig. 1); a de ureia em $20 \mathrm{mg}$ e $40 \mathrm{mg}$ (Fig. 2); a de ácido úrico em $100 \mathrm{mg}$ (Fig. 3) e a de creatinina em 20 $\mathrm{mg}$ e $80 \mathrm{mg}$ de $\mathrm{CaCO}_{3}$, todas as comparações em relação ao controle (Fig. 4). Em B. glabrata, as maiores concentrações de proteína totais foram observadas nas exposições a $60 \mathrm{mg}, 80 \mathrm{mg}$ e 100 $\mathrm{mg}$ de $\mathrm{CaCO}_{3}$ (Fig. 1); ureia no grupo controle (Fig. 2); ácido úrico em $80 \mathrm{mg}$ e creatinina em $40 \mathrm{mg}$ de $\mathrm{CaCO}_{3}$ (Fig. 4), todas as comparações em relação ao controle.

Tab. 1. Valores de F e P para a análise de variância de acordo com o tipo de produto do metabolismo e espécie avaliada. Aqui foram considerados significativos valores de $P<0,01$ seguindo o critério Bonferroni para múltiplos testes (ver detalhes nos métodos). Em todas as Anovas, o grau de liberdade do tratamento foi igual a 5 e o grau de liberdade do resíduo igual a 12 . SQ = soma dos quadrados; $\mathrm{QM}=$ quadrado médio.

\begin{tabular}{|c|c|c|c|c|c|c|c|c|}
\hline \multirow[t]{2}{*}{ Produto } & \multicolumn{4}{|c|}{ Biomphalaria tenagophila } & \multicolumn{4}{|c|}{ Biomphalaria glabrata } \\
\hline & SQ & QM & $\mathrm{F}$ & $\mathrm{P}$ & SQ & QM & $\mathrm{F}$ & $\mathrm{P}$ \\
\hline Proteínas & 2,1 & 0,4 & 31,1 & $<0,001$ & 52,5 & 10,5 & 14,8 & $<0,001$ \\
\hline Ureia & 452 & 90 & 149,8 & $<0,001$ & 369 & 73 & 102,3 & $<0,001$ \\
\hline Ácido Úrico & 23 & 4,6 & 118,4 & $<0,001$ & 9,6 & 1,9 & 27,0 & $<0,001$ \\
\hline Creatinina & 0,6 & 0,1 & 102,2 & $<0,001$ & 0,3 & 0,06 & 102,5 & $<0,001$ \\
\hline
\end{tabular}

Rev. Biol. Neotrop. / J. Neotrop. Biol., Goiânia, v. 16, n. 2, p. 61-69, jul.-dez. 2019 


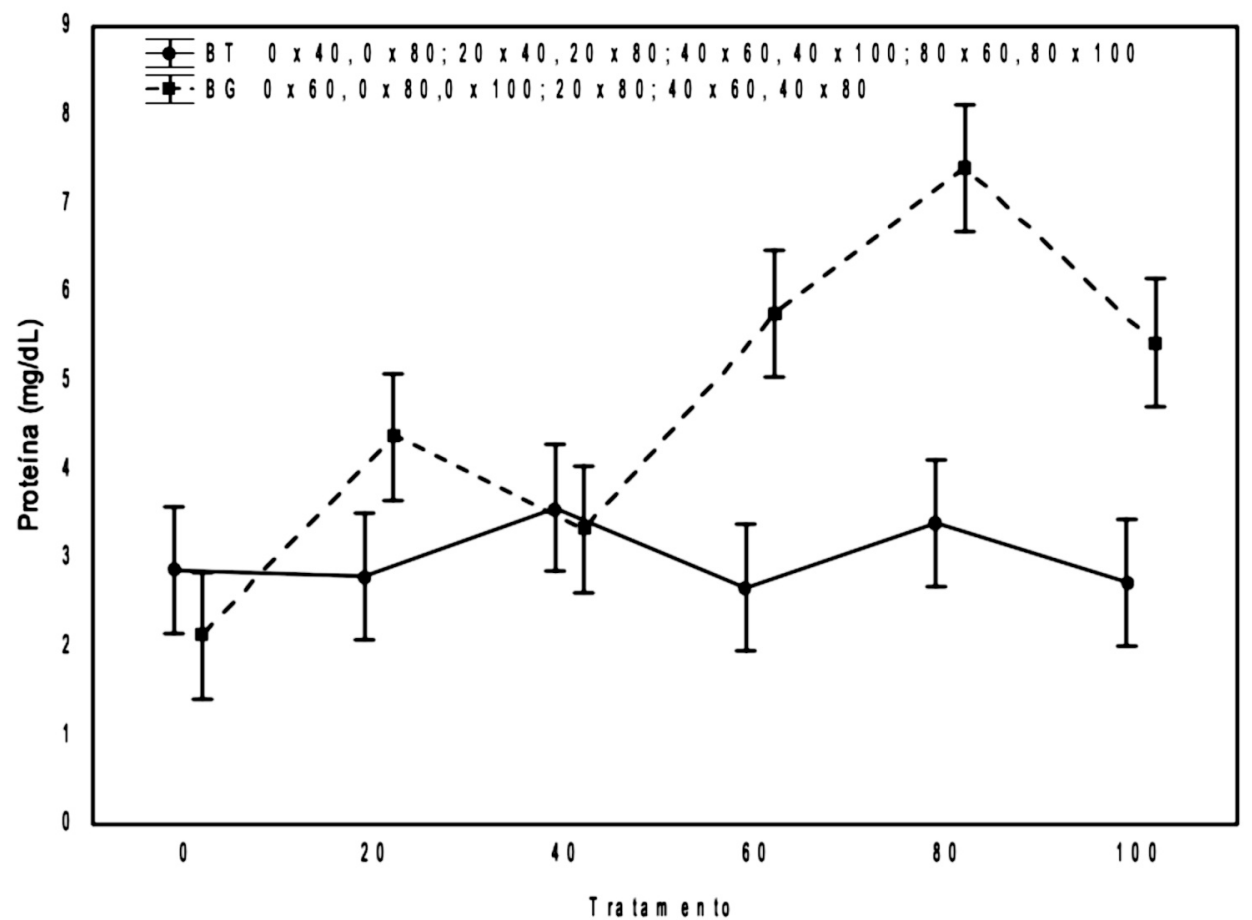

Fig. 1. Média e intervalos de confiança (95\%) para as concentrações de proteínas, nas espécies Biomphalaria tenagophila (BT) e Biomphalaria glabrata (BG) expostas a diferentes concentrações de $\mathrm{CaCO}_{3}$. As comparações indicam entre quais tratamentos foram encontradas diferenças significativas para o teste de Tukey $(\mathrm{P}<0,05)$.

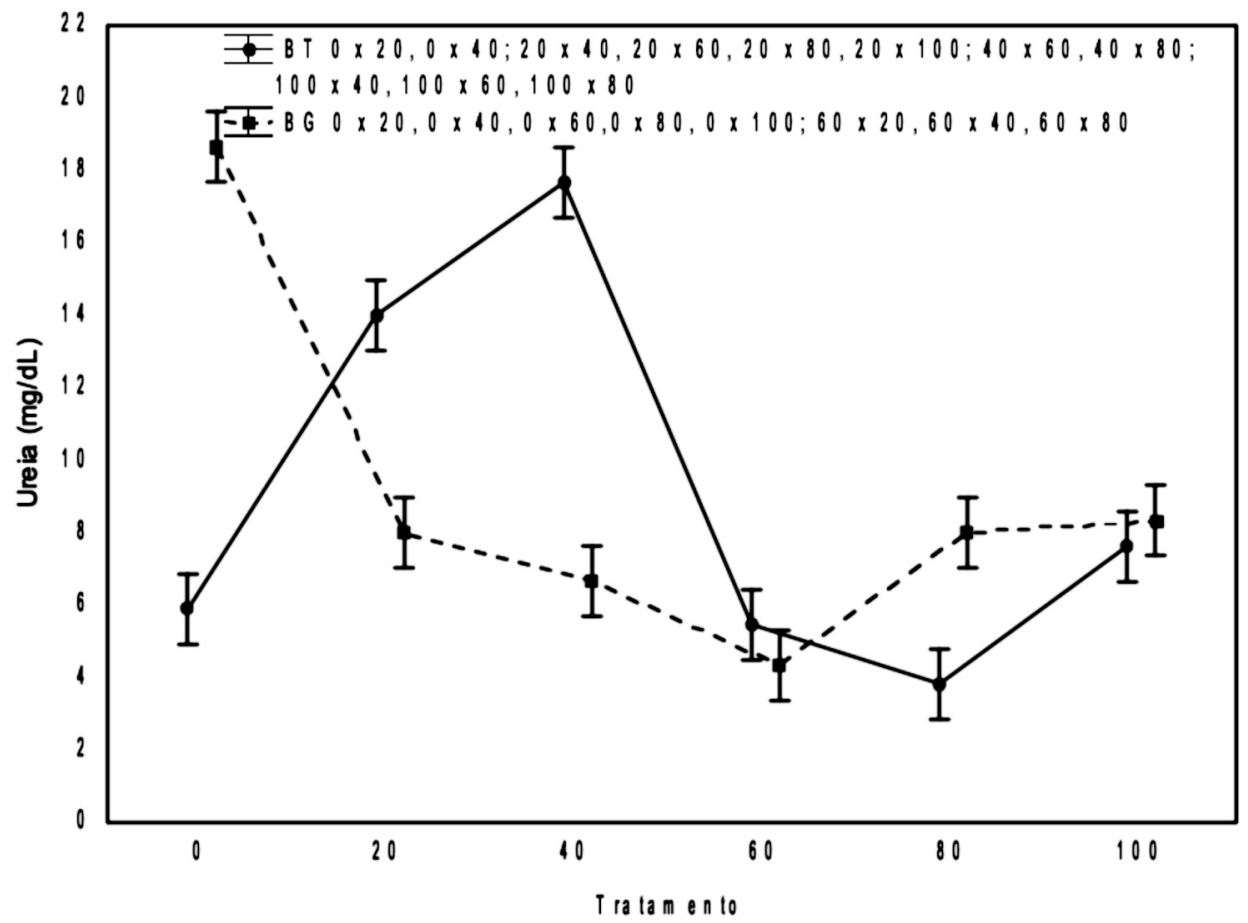

Fig. 2. Média e intervalos de confiança (95\%) para as concentrações de ureia nas espécies Biomphalaria tenagophila (BT) e Biomphalaria glabrata (BG) expostas a diferentes concentrações de $\mathrm{CaCO}_{3}$. As comparações indicam entre quais tratamentos foram encontradas diferenças significativas para o teste de Tukey $(\mathrm{P}<0,05)$. 


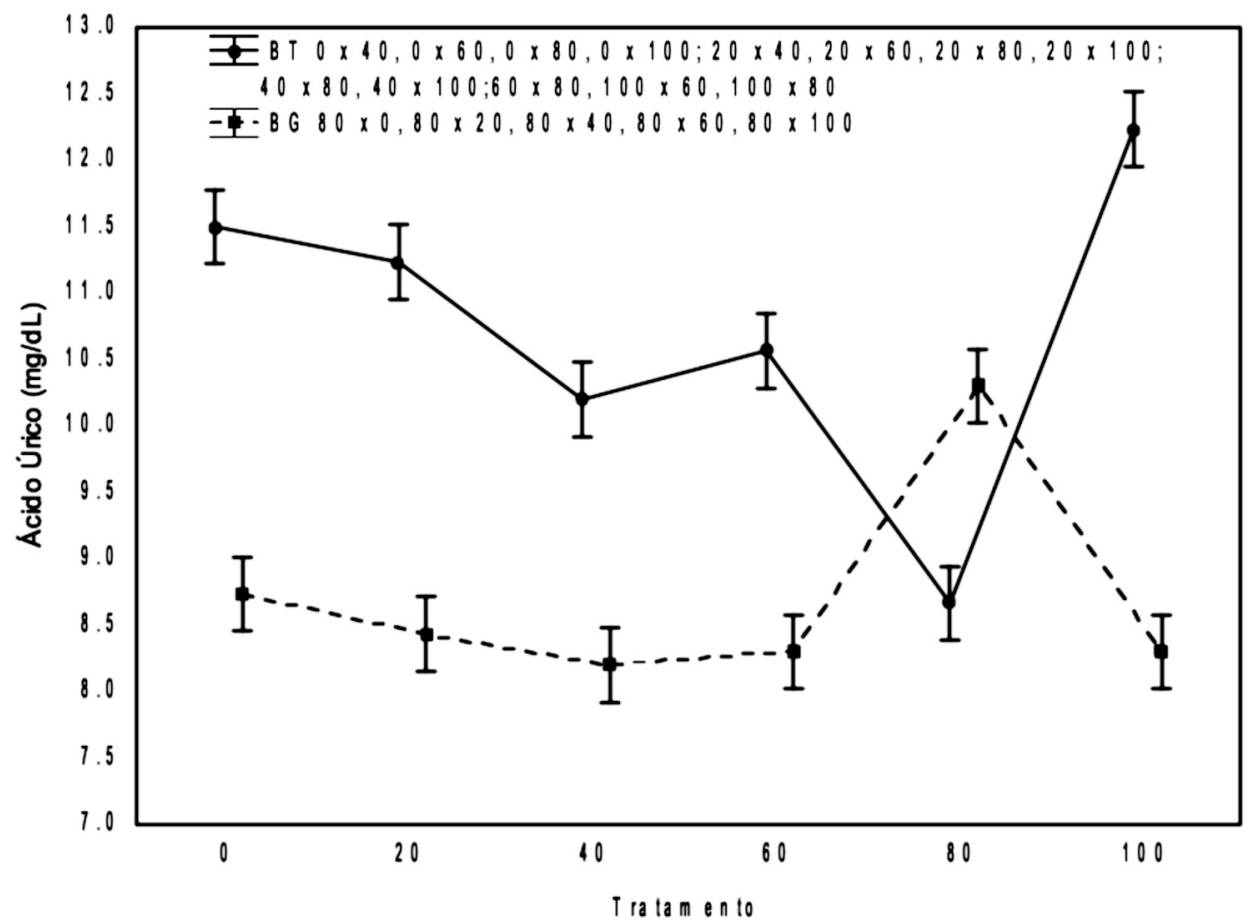

Fig. 3. Média e intervalos de confiança (95\%) para as concentrações de ácido úrico nas espécies Biomphalaria tenagophila (BT) e Biomphalaria glabrata (BG) expostas a diferentes concentrações de $\mathrm{CaCO}_{3}$. As comparações indicam entre quais tratamentos foram encontradas diferenças significativas para o teste de Tukey $(\mathrm{P}<0,05)$.

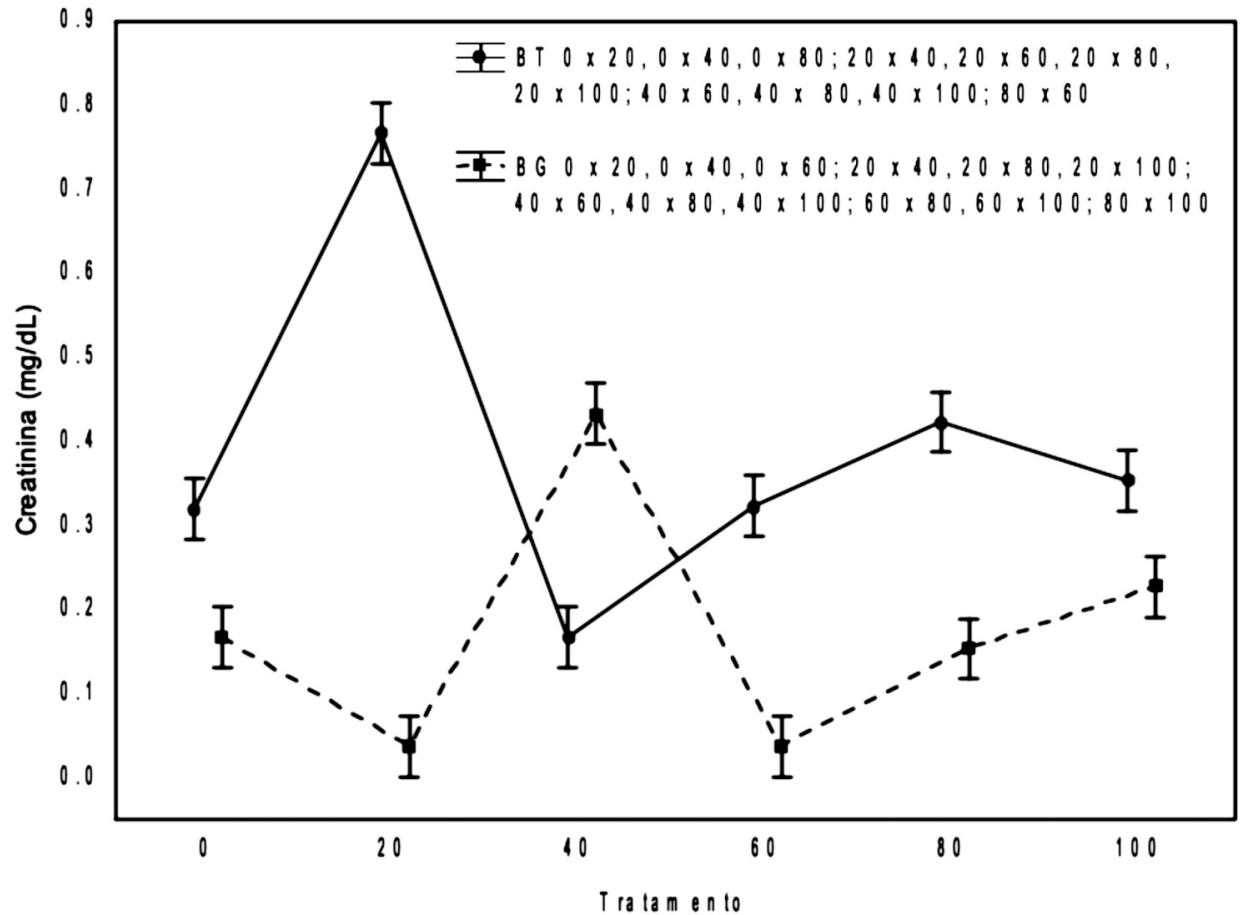

Fig. 4. Média e intervalos de confiança (95\%) para as concentrações de creatinina nas espécies Biomphalaria tenagophila (BT) e Biomphalaria glabrata (BG) expostas a diferentes concentrações de $\mathrm{CaCO}_{3}$. As comparações indicam entre quais tratamentos foram encontradas diferenças significativas para o teste de Tukey $(\mathrm{P}<0,05)$. 
Para B. tenagophila, nas comparações entre concentrações de $\mathrm{CaCO}_{3}$, houve aumento de proteínas totais nas exposições a 40 e $80 \mathrm{mg}$ comparadas a $20 \mathrm{mg}$; enquanto, nas comparações entre 60 e $100 \mathrm{mg}$ x $40 \mathrm{mg}, 80$ × $60 \mathrm{mg}$, e $100 \times 80 \mathrm{mg}$ a concentração de proteínas totais diminui nas exposições as menores concentrações de $\mathrm{CaCO}_{3}$. Em B. glabrata, as comparações entre concentrações de $\mathrm{CaCO}_{3}, 80 \times 20,60$ e 80 x $40 \mathrm{mg}$ apontaram para aumento nas concentrações de proteínas totais na hemolinfa dos caramujos a maiores concentrações de $\mathrm{CaCO}_{3}$.

$\mathrm{Na}$ comparação entre espécies, observouse que a exposição de $B$. tenagophila ao $\mathrm{CaCO}_{3}$ resultou em menores valores de proteínas totais e maiores valores de ureia, ácido úrico e creatinina (Fig. 1, 2, 3 e 4).

$O$ déficit de carboidratos faz com que os caramujos utilizem substratos alternativos para obter a energia necessária aos seus processos metabólicos vitais (Pinheiro et al., 2009). O uso de proteínas como substrato energético, gera alterações na concentração destas, assim como, de aminoácidos livres no caramujo (Becker, 1980).

A ativação do catabolismo proteico é sugerido, quando ocorre um significativo decréssimo de proteínas totais na hemolinfa e glândula digestiva, principal tecido de armazenamento deste substrato", do caramujo (Pinheiro et al., 2009). Nesse sentido, a exposição ao $\mathrm{CaCO}_{3}$ levou ao catabolismo proteico nas duas espécies avaliadas, sendo que em $B$. tenagophila a utilização de proteínas ocorreu nas exposições a $20 \mathrm{mg}$ e $100 \mathrm{mg}$ de $\mathrm{CaCO}_{3}$, ou seja, na menor e maior concentração avaliada, o que sugere um valor máximo de cálcio para manutenção da homeostase, sendo este $80 \mathrm{mg}$. Em B. glabrata a utilização de proteínas ocorreu nas exposições a 20 e $40 \mathrm{mg}$ de $\mathrm{CaCO}_{3}$, reforçando que onde o cálcio é limitado a menos de $50 \mathrm{mg} / \mathrm{L}$, ocorre maior gasto de energia (Dalesman et al., 2011).

Dados semelhantes foram observados para menores valores de glicose na hemolinfa de $B$. glabrata exposta, por 30 dias, a 20 e $40 \mathrm{mg}$ $\mathrm{CaCO}_{3}$. A utilização de glicose como substrato energético foi comprovada pela ativação do ciclo do ácido tricarboxilíco (ATC) pelo aumento nas concentrações dos ácidos orgânicos piruvato, oxaloacetato, citrato, succinato e fumarato (Silva et al., 2017). O fumarato pode surgir, ainda, via ciclo arginina-ornitina-ureia, a partir de reações enzimáticas. Um segundo grupo amido, originado do aspartato, é adicionado à citrulina, formando argininosuccinato, o qual é degradado por uma argininosuccinase, liberando arginina e fumarato.

Quando utilizados como fonte energética, os aminoácidos são desmembrados em um esqueleto carbônico, que segue como um $\alpha$ cetoácido, e em um grupo $\mathrm{NH}_{4}{ }^{+}$. A amônia produzida neste processo pode ser destinada à formação de novos aminoácidos, aminas biológi- cas e nucleotídeos; ou ainda ser desviada para formação de carbamil fosfato e entrar no ciclo da ornitina. Caso o grupamento $\mathrm{NH}_{4}{ }^{+}$ seja endereçado para formação de nucleotídeos, esta síntese ocorre pela via das purinas, tendo como um dos seus produtos o ácido úrico. Porém, se o destino for à formação de carbamil fosfato, o grupamento nitrogenado entra no ciclo da ornitina e é excretado na forma de ureia (Nelson \& Cox, 2011).

A ureia como produto do catabolismo proteico foi demonstrada em $B$. tenagophila exposta a $20 \mathrm{mg}$ de $\mathrm{CaCO}_{3}$ e no grupo controle de $B$. glabrata, através do aumento deste produto na hemolinfa das duas espécies. $O$ aumento na concentração de creatinina por $B$. tenagophila exposta a $20 \mathrm{mg}$ e $80 \mathrm{mg}$ e $B$. glabrata a $40 \mathrm{mg}$ de $\mathrm{CaCO}_{3}$ foi interpretado como produto final do ciclo da ureia (Cian et al., 2000). O aumento na concentração de ácido úrico foi observado em $B$. tenagophila exposta a $100 \mathrm{mg}$ de $\mathrm{CaCO}_{3}$ e em B. glabrata a $80 \mathrm{mg}$ de $\mathrm{CaCO}_{3}$.

O ácido úrico excretado por moluscos aquáticos apresenta a vantagem de ter pouca perda de água vinculada a sua produção. A via metabólica pela qual os moluscos podem detoxificar seus produtos nitrogenados de excreção pode variar de acordo com o habitat por ele ocupado. Desta forma, dependendo dos estímulos do meio ou de alguma situação de estresse, um molusco que vive em um ambiente aquático pode favorecer a excreção de ureia, uma vez que sua eliminação dispõe de significativa perda de água, porém com menor gasto energético, atrelado ao menor consumo de NADPH. As inversões do perfil uricotélico para ureotélico podem estar vinculadas a uma vasta lista de razões (Tunholi et al., 2011; Pinheiro et al., 2009; Becker 1980). Dessa forma, estudos sobre o metabolismo de moluscos Biomphalaria auxilia na compreensão da relação parasito-hospedeiro, tendo em vista que a espécie $B$. tenagophila é menos suceptível à infecção por $S$. mansoni comparada a B. glabrata.

\section{REFERÊNCIAS}

Allen, P. J., K. E. D'Anci \& R. B. Kanarek. 2011. Creatine, brain functioning, and behavior. pp. 215-236. In: Kanarek, R. B. \& H. R. Lieberman. (Ed.). Diet, Brain and Behavior: Practical Implications. CRC Press Book.

Barbosa, F. S. \& S. C. Barbosa. 1994. The bioecology of snail vectors for Schistosomiasis in Brazil. Cad. Saúde Públ. 10: 200-209. 
Becker, W. 1980. Metabolic interrelation ships of parasitic trematodes and mollusks, especially Schistosoma mansoni in Biomphalaria glabrata. Z. Parasitenkd. 63: 101-111.

Cian, M. C., M. Regnault \& F. H. Lallier. 2000. Nitrogen metabolites and related enzymatic activities in the body fluids and tissues of the hydrothermal vent tubeworm Riftia pachyptila. J. Exp. Biol. 203: 2907-2920.

Core Team R. 2016. R: A language and environmental for statistical computing. Vienna, Austria: $\mathrm{R}$ Foundation for Statistical Computing.

Dalesman, S., M. H. Braun \& K. Lukowiak. 2011. Low environmental calcium blocks long term memory formation in a pulmonate snail. Neurobiol. Learn. Mem. 95: 393-403.

Davies, T. W. \& D. A. Erasmus. 1984. An ultrastructural study of the effect of parasitism by larval Schistosoma mansoni on the calcium reserves of host, Biomphalaria glabrata. Cell. Tissue. Res. 236: 643-649.

Dunker, W. 1848. Diagnoses specierum novarum generis Planorbis collectionis Cumingianae. Proc. Zool. Soc. London. 16: $40-43$.

Elbaz, T. \& G. Esmat. 2013. Hepatic and Intestinal Schistosomiasis: Review. J. Adv. Res. 4: 445-452.

Gray, D. J., D. P. McManus, Y. Li, G. M. Williams, R. Bergquist \& A. G. Ross. 2010. Schistosomiasis elimination: lessons from the past guide the future. Lancet. Infect. Dis. 10: 733-736.

Greenaway, P. 1971. Calcium regulation in the freshwater mollusc, Limnaea stagnalis (L.) (Gastropoda: Pulmonata). I. The effect of internal and external calcium concentrations. J. Exp. Biol. 54: 199-214.

Henry, J. B. 1996. Clinical diagnosis and management by laboratory methods. 19nd ed. Philadelphia: Saunders.

Hiong, K. C., A. M. Loong, S. F. Chew \& Y. I. Kwong. 2005 Increases in urea synthesis and the ornithine-urea cycle capacity in the giant african snail, Achatina fulica, during fasting or aestivation, or after the injection with ammonium chloride. J. Exp. Zool. 303: 1040-1053.

Joosse, J. 1988. The hormone molluscs. pp. 89140. In: H. Janfer \& R. G. H. Dwner (Ed.). Vertebrate Endocrinology. Academic Press.

King, C. H. \& D. Bertsch. 2015 Historical Perspective: Snail Control to Prevent Schistosomiasis. Plos Negl. Trop. Dis. 9: 16.

Lardans, V. \& C. Dissous. 1998. Snail control strategies for reduction of schistosomiasis transmission. Parasitol. Today. 14: 413-417.

Legendre, P. \& L. Legendre. 1998. Numerical Ecology. 2nd ed. Amsterdam: Elsevier Science.

Liebsch, M. \& W. Becker. 1990. Comparative glucose tolerance studies in the freshwater snail Biomphalaria glabrata: influence of starvation and infection with the trematode Schistosoma mansoni. J. Comp. Physiol. B. 160: 41-50.

Livingstone, D. R. \& A. de Zwaan. 1983. Carbohydrate metabolism of gastropods. pp. 177-242. In: Hochachka, P. W. (Ed.). The Mollusca Metabolic Biochemistry and Molecular Biomechanics. Academic Press.

Nelson, D. L. \& M. M. Cox. 2011. Princípios de Bioquímica de Lehninger. Porto Alegre: Artmed.

Oksanen, J., F. G. Blanchet, M. Friendly, R. Kindt, P. Legendre, D. McGlinn, P. R. Minchin, R. B. O'Hara, G. L. Simpson, P. Solymos, M. H. H. Stevens, E. Szoecs \& H. Wagner. 2017. Vegan: Community Ecology Package. R. Package. Version. 2.44.

Orbigny, A. 1835. Synopsis terrestrium et fluviatilium molluscorum, in suo per Americam meridionalem itinere collectorum. Mag. Zool. 5: 1-44.

Paraense, W. L. 2001. The schistosome vectors in the Americas. Mem. Inst. Oswaldo Cruz. 96: 7-16.

Pinheiro, J., A. JR. Maldonado \& R. M. Lanfredi. 2009. Physiological changes in Lymnaea columella (Say, 1818) (Mollusca, Gastropoda) in response to Echinostoma paraensei Lie and Basch, 1967 (Trematoda, Echinostomatidae) infection. Parasitol. Res. 106: 55-59. 
Sambon, L. W. 1907. Descriptions of some new species of animal parasites. Proc. Zool. Soc. London. 19: 282-283.

Say, T. 1818. Account of two new genera, and several new species, of fresh water and land shells. J. Acad. Nat. Sci. Phila. 1: 276-284.

Scholte, R. G. C., O. S. Carvalho, J. B. Malone, J. Utzinger \& P. Vounatsou. 2012. Spatial distribution of Biomphalaria spp., the intermediate host snails of Schistosoma mansoni, in Brazil. Geospat. Health. 6: 95-101.

Silva, L. D., V. C. S. Amaral, M. C. Vinaud, A. M. Castro, H. H. A. Rezende, D. B. Santos, C. C. Mello-Silva \& J. C. B. Bezerra. 2017. Changes in energetic metabolism of Biomphalaria glabrata (Mollusca, Planorbidae) in response to exogenous calcium. Braz. J. Biol. 77: 304311.

Tunholi, V. M., D. Lustrino, V. M. TunholiAlves, C. C. C. Mello-Silva, A. JR. Maldonado, M. L. A. Rodrigues \& J. Pinheiro. 2010. Changes in the reproductive biology of Biomphalaria glabrata infected with different doses of Echinostoma paraensei miracidia. J. Invertebr. Pathol. 106: 192-195.

Tunholi, V. M., D. Lustrino, V. M. TunholiAlves, J. S. Garcia, C. C. C. Mello-Silva, A. JR. Maldonado, M. L. A. Rodrigues \& J. Pinheiro. 2011. Influence of Echinostoma paraensei (Lie and Basch, 1967) infection on the calcium content in Biomphalaria glabrata (Say, 1818). Exp. Parasitol. 129: 266-269.

World Health Organization. 2012. Schistosomiasis: population requiring preventive chemotherapy and number of people treated in 2010. Wkly. Epidemiol. Rec. 4: 37-44. 